\title{
Towards Continuous Primary Manufacturing Processes- Particle Design through Combined Crystallization and Particle Isolation
}

\author{
Claas Steenweg ${ }^{+}$(D) Anne Cathrine Kufner ${ }^{\dagger}$, Jonas Habicht and Kerstin Wohlgemuth *(D) \\ Department of Biochemical and Chemical Engineering, Laboratory of Plant and Process Design, \\ TU Dortmund University, D-44227 Dortmund, Germany; claas.steenweg@tu-dortmund.de (C.S.); \\ anne.kufner@tu-dortmund.de (A.C.K.); jonas.habicht@tu-dortmund.de (J.H.) \\ * Correspondence: kerstin.wohlgemuth@tu-dortmund.de; Tel.: +49-(0)-231-755-3020 \\ + Claas Steenweg, Anne Cathrine Kufner contributed equally to this work.
}

check for updates

Citation: Steenweg, C.; Kufner, A.C.; Habicht, J.; Wohlgemuth, K. Towards Continuous Primary Manufacturing Processes-Particle Design through Combined Crystallization and Particle Isolation. Processes 2021, 9, 2187. https://doi.org/10.3390/ pr9122187

Academic Editor: Dimitrios

I. Gerogiorgis

Received: 30 September 2021 Accepted: 27 November 2021 Published: 3 December 2021

Publisher's Note: MDPI stays neutral with regard to jurisdictional claims in published maps and institutional affiliations.

Copyright: (c) 2021 by the authors. Licensee MDPI, Basel, Switzerland. This article is an open access article distributed under the terms and conditions of the Creative Commons Attribution (CC BY) license (https:/ / creativecommons.org/licenses/by/ $4.0 /)$.

\begin{abstract}
Integrated continuous manufacturing processes of active pharmaceutical ingredients (API) provide key benefits concerning product quality control, scale-up capability, and a reduced time-tomarket. Thereby, the crystallization step, which is used in approximately $90 \%$ of API productions, mainly defines the final API properties. This study focuses on the design and operation of an integrated small-scale process combining a continuous slug flow crystallizer (SFC) with continuous particle isolation using the modular continuous vacuum screw filter (CVSF). By selective adjustment of supersaturation and undersaturation, the otherwise usual blocking could be successfully avoided in both apparatuses. It was shown that, during crystallization in an SFC, a significant crystal growth of particles $\left(\Delta d_{50,3} \approx 220 \mu \mathrm{m}\right)$ is achieved, and that, during product isolation in the CVSF, the overall particle size distribution (PSD) is maintained. The residual moistures for the integrated process ranged around $2 \%$ during all experiments performed, ensuring free-flowing particles at the CVSF outlet. In summary, the integrated setup offers unique features, such as its enhanced product quality control and fast start-up behavior, providing a promising concept for integrated continuous primary manufacturing processes of APIs.
\end{abstract}

Keywords: continuous manufacturing; pharmaceutical manufacturing; continuous crystallization; continuous particle isolation; filtration; washing; particle design; particle size distribution

\section{Introduction}

Using crystallization as a separation step during downstream processing of manufacturing of active pharmaceutical ingredients (API) is a common approach to perform product design in the pharmaceutical industry. The preferred formulation of APIs is usually crystalline as solid dosage form, and water solubility of APIs is often low, resulting in poor bioavailability [1]. Crystallization processes provide some key benefits compared to other separation techniques, namely high product purity and adjustable particular product properties [2]. Therefore, in more than $90 \%$ of API production pathways, one or even more crystallization steps are involved [3]. Having formed the key compound in a chemical or biotechnological reaction, crystallization is used to create a solid phase containing preferentially pure API in the crystal form desired. Crystallization directly influences the final product purity and should therefore be optimized to ensure both consistent purity and particle size distribution (PSD) [4,5]. Upcoming challenges are the solid-liquid separation as well as washing and drying steps to meet specific product specifications regarding residual moisture and preservation of PSD [6]. Although pharmaceutical manufacturing has been traditionally employed in batch mode, continuous processes possess some crucial benefits, such as eliminating batch-to-batch-variability or reducing time-to-market due to an easier scale-up $[7,8]$. The overall goal is to create an end-to-end small-scale continuous manufacturing process for the typical API production scale of $250-1000 \mathrm{~kg}$ per 
year, starting from raw material treatment to final dosage form [9]. Thus, new challenges establish including the development of robust continuous synthesis, crystallization as well as product isolation and formulation in the pharmaceutical industry under defined product quality restrictions [5]. A number of recent review papers illustrate the fundamentals and challenges of individual unit operations (e.g., crystallization) $[9,10]$ and also of combined and integrated processes $[4,5,8]$.

To realize the advantages of continuous manufacturing, various approaches for smallscale continuous crystallization concepts have been made. Two main classes of continuous crystallization devices relating to mixed-suspension mixed-product removal crystallizers (MSMPR) and tubular plug-flow crystallizers (PFC) can be distinguished [4,5,8,9]. During this work, a special type of PFC, the slug flow crystallizer (SFC), was used, which is a promising apparatus with regard to the generation of desired product crystals and high yield in a reproducible manner. The SFC is characterized by its segmented flow of two immiscible fluids, ensuring no back-mixing despite laminar flow conditions compared to the operation of tubular crystallizers having only one fluid. Consequently, a narrow residence time distribution (RTD) was obtained [11], which is a precondition for the maintenance of a narrow PSD. Internal vortices were induced, caused by the wall's no-slip condition, ensuring particles' suspension in the slugs without the use of an external device. This resulted in a gentle treatment of the particles as attrition and secondary nucleation are reduced. To enable the kinetically limited crystal growth to the desired product size, the residence time can be adjusted flexible via the volume flow rate, as well as the tubing length. In order to simplify the following product isolation, it is advisable to use gas-liquid segmented flow to avoid liquid-liquid separation in further downstream and thus ensure high product purity. Hence, in this study a gaseous phase was used as segmentation medium to delineate the liquid slugs. For more information about the research on the SFC, the reader is referred to the literature. $[9,10,12-14]$

Afterwards, preserving the produced critical quality attributes (CQAs) was a crucial requirement for all subsequent isolation steps, namely filtration, washing, and drying. To fulfill this separation task continuously, different approaches were used and discussed in detail in our previous publications [6,15]. The approach of automated batch filtration with cycle times using the continuous filter carousel (CFC) was developed by Alconbury Weston Ltd. The characterization of the CFC was first performed by ACEVEDO ET AL. with regard to final residual moisture reduction [16]. In further studies carried out by DОМОКОS ET AL., NAGY ET AL., and LIU ET AL., emphasis was put on a systematic analysis of the relations between operational parameters and CQAs [17-19]. The second approach of creating a full-continuous apparatus for solid-liquid separation was introduced by MASCIA ET AL. [20]. With the overall goal to develop end-to-end continuous manufacturing of APIs, they invented the continuous rotary plate filter (CRPF) for solid-liquid separation and washing. In the recent studies of TESTA ET AL. and HU ET AL., the CRPF was used for isolation after reactive crystallization of acetaminophen from ethanol and acetylic salicylic acid from an alcoholic mixture, respectively $[7,21]$. Here, emphasis was put on the integrated design of creating an end-to-end manufacturing process. The crystals leaving the CRPF were either resuspended or reduced in size by a high-shear mixer $[7,21]$.

To efficiently perform all necessary product isolation steps after a preceding continuous crystallization, the continuous vacuum screw filter (CVSF) was developed and used in this work [22]. The CVSF was designed for feed suspension volume flow rates of $10-100 \mathrm{~mL} \mathrm{~min}^{-1}$ [6]. This novel apparatus can be used in flexible modular connections of filtration, washing, and drying modules $[6,15,23]$. The tubular glass body of the CVSF was directly fed with suspension leaving the crystallizer so that solid-liquid separation is realized by an applied vacuum, with the help of a glass filter frit in the first module. A rotating screw axially transports the remaining solid particles to the following modules in a series connection. Having performed the subsequent washing and additional drying tasks, the overall goal is to discharge free-flowing crystals with no significant change in the PSD. The first characterization was performed with a well-filterable model suspension 
consisting of L-alanine particles resuspended in saturated aqueous solution $[6,15]$. For this system and suspension volume flow rates of up to $84 \mathrm{~mL} \mathrm{~min}^{-1}$ and corresponding solid contents $w_{S}$ of $6 \mathrm{wt} . \%$, suitable residual moistures of $1 \%$ with a preserved PSD were obtained using filtration and a two-stage washing [15]. Moreover, a narrow residence time distribution of the solid phase $\left(\mathrm{RTD}_{\mathrm{s}}\right)$ was defined as a fundamental requirement and thus, systematically proved in our previous works $[6,15]$.

The detailed characterization of continuous downstream apparatuses (crystallization, product isolation) is a crucial requirement to meet high product quality specifications reliably. These specifications are only achievable by having a detailed understanding of the relations between input and output parameters [4]. Thus, the characterization of apparatuses is often performed separately. However, in combining two or more apparatuses in an integrated process, even more complex relationships arise [4,8]. Especially for crystallization and filtration, the influencing parameters are highly interconnected, and the combined effects are complex [6]. Therefore, the definition of CQAs and the systematic manipulation by varied operating parameters is the main part of the quality-by-design $(\mathrm{QbD})$ approach [8]. Regarding filtration performance, filter clogging is reported in several cases as a challenge of connecting a continuous crystallization with a solid-liquid separation step [4,16-19]. This phenomenon was not quantified towards potential influence factors (fine fraction, supersaturation) affecting the clogging of the apparatuses used. In the case of a MSMPR-CFC connection, filter clogging, was overcome by a cleaning-in-place (CIP) or dissolution of the clogging particles [19]. This strategy results in a loss of product and a cleaning time where no suspension is fed to CFC. Thus, a reliable concept to prevent filter clogging caused by the preceding crystallization would be favorable and is an explicit demand from industry [5].

This study aims at the design and operation of a full continuous process combining SFC with subsequent product isolation using CVSF. The following requirements are addressed and fulfilled:

1. Enabling an integrated process and ensuring continuous operability

2. Designing CQAs during crystallization and preserving them during particle isolation We hypothesize the following:

1. The connection of the two apparatuses is only possible in a stable manner if supersaturation is specifically reduced before entering the CVSF

2. Clogging of the two apparatuses can be prevented by specific process control, so that long-term operation is possible

\section{Plant Design and Integrated Setup from Solution to Solid Product}

A main difference between batch and continuous crystallization processes is that at the end of a continuous crystallizer the thermodynamic equilibrium is not achieved, and the remaining supersaturation in the filtration can quickly lead to clogging of the filter media. To the best of our knowledge, no systematic study has been conducted so far on controlled supersaturation degradation after small-scale continuous crystallization processes. An exemplary c,T-diagram is shown in Figure 1. During the cooling crystallization process (Point $1 \rightarrow$ Point 2 ) a supersaturation level is always present to ensure a certain process yield. In order to reduce this residual supersaturation, three methods are suitable: The first option (a) is the implementation of an isothermal holding section in the continuous crystallizer (comparable to holding time in batch). In this case, however, it is never possible to cross the solubility curve into the undersaturated state, which could improve the longterm operability of the filter. Furthermore, this would increase the residence time, reduce the space-time-yield and increase the investment costs. The second option (b) is dilution with pure solvent. However, this does not increase the yield, since only the mass of solvent is increased. In addition, there is a risk of impurities being introduced into the system. The third option (c) is rapid heating. For this purpose, an additional heater is required, which enables a precise adjustment of the saturation state, or respectively the level of undersaturation. This is the most promising variant, since operation of the particle 
isolation apparatus is possible regardless of the final temperature of the crystallizer and, in addition, only low investment and operating costs are incurred.



Figure 1. Concepts to realize degradation of supersaturation in continuous cooling crystallization. Point 1 is the start of the crystallization (nucleation) and Point 2 is the end of the continuous crystallizer.

Due to the concept of rapid heating after the crystallizer in order to eliminate the residual supersaturation present, the realization of a continuous small-scale manufacturing process consisting of crystallization and subsequent product isolation is possible. Therefore, the experimental setup shown in Figure 2 was used. The applied process can be divided into three sections: In section I, continuous cooling crystallization took place in order to adjust the product particle properties. The product particles obtained and grown in the crystallizer were continuously conveyed through a heater in section II, which reduces the residual supersaturation still present in the solution. Afterwards, the product suspension was continuously filtered, washed and dried. The various sections are briefly presented below.

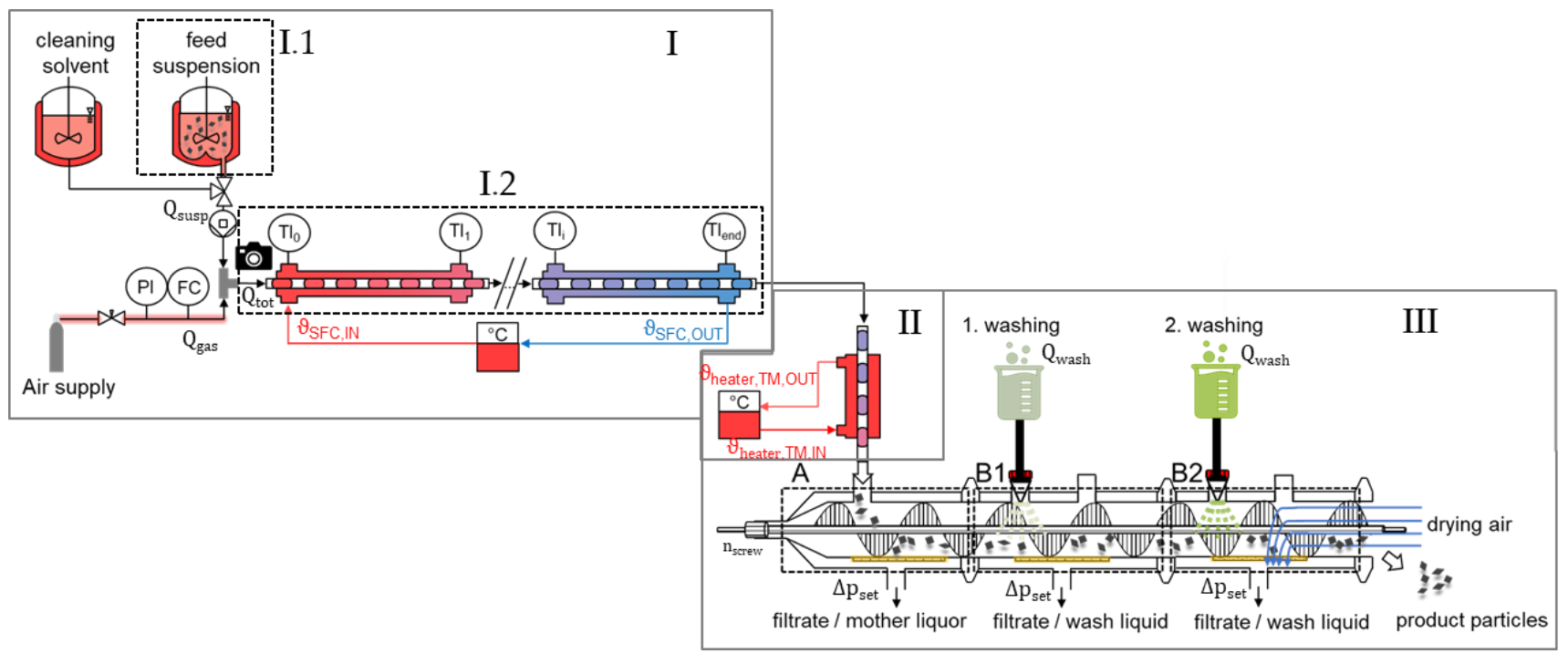

Figure 2. Schematic experimental setup for continuous small-scale manufacturing consisting of a slug flow crystallizer (SFC, (I)), a heater (II) and the continuous vacuum screw filter (CVSF, (III)). A corresponding photograph is given in the supplementary materials in Figure S1. 
In section I the continuous cooling crystallization is performed in an SFC. Therefore, inside the nucleation zone (section I.1), seed crystals were added into a saturated solution $(V=450 \mathrm{~mL})$. In order to guarantee a homogeneous crystal suspension, a stirrer was used and the vessel's bottom was designed conical according to the design procedure of LÜHRMANN ET AL. [24]. Afterwards, the feed suspension was transported vertically via a peristaltic pump (Ismatec Reglo Digital MS-4/12, $d_{i}=2.29 \mathrm{~mm}$ Pharmed) to a T-junction (polypropylene, PP). At the T-junction $\left(d_{i}=3 \mathrm{~mm}\right)$, hydrodynamic slug formation was carried out by a squeezing mechanism, forming alternating liquid and gas segments of equal size. The gas volume flow rate was supplied via pipeline pressure from a gas cylinder and manually controlled by a high-resolution needle valve (NV-001-HR, Bronkhorst) and a flow meter (El-Flow-Select, Bronkhorst). [25]

The liquid segments contain the seed crystals, which were intended to grow inside the growth zone of the apparatus (section I.2). The growth zone consisted of a fluorinatedethylene-propylene (FEP) tubing of a length of $L_{\text {tubing }}=26 \mathrm{~m}$ and an inner diameter of $d_{i}=3.18 \mathrm{~mm}\left(d_{\text {out }}=4.76 \mathrm{~mm}\right)$. By choosing a hydrophobic wall material for the application of an aqueous substance system, the absence of a wall film was realized, caused by the high contact angle $\theta_{\mathrm{FEP}, \mathrm{H} 2 \mathrm{O} \text {,air }}$ of the tubing's material [26]. As a result, convex slugs were formed and a narrow RTD of the solid and liquid phases could be ensured $[11,26]$. The driving force is adjusted by cooling the suspension with the aid of a tube-in-tube design operated in co-current mode using a thermostat (Huber CC-K6). Therefore, deionized water as tempering medium up to $50^{\circ} \mathrm{C}$ was utilized in an outer polyvinyl chloride (PVC) cooling jacket $\left(d_{i}=15 \mathrm{~mm}\right)$ covering the SFC process tubing. This temperature cooling concept enabled a smooth temperature profile along the process tubing, avoiding supersaturation peaks. Further details on the cooling concept and SFC design were already published in our previous studies $[11,25]$.

In section II, a vertical installed tube-in-tube heater between SFC and continuous product isolation was implemented to realize degradation of supersaturation by simultaneously ensuring particle suspension. The idea of this concept is an elimination of the supersaturation to prevent filter clogging, with the help of rapidly heating the suspension to avoid the dissolution of the particles caused by the heater and thus inhibit changes in PSD. Therefore, a small heating area $\left(\approx 30 \mathrm{~cm}^{2}\right)$ was chosen to reduce the residence time $\left(\tau_{\text {heater }}\right)$ of the suspension in the heating section, which was realized by a counter-current tube-in-tube heater made of FEP (inner tube for process medium) and PVC (outer tube for tempering medium). Relatively high tempering medium (deionized water) temperatures of up to $75^{\circ} \mathrm{C}$ were needed to control the temperature by the thermostat (Huber CC-K6). By virtue of the low $\tau_{\text {heater }}(<10 \mathrm{~s})$ for the desired experimental suspension flow rates, changes in PSD were not expected, which was validated in preliminary experiments (Section 4.1.1). The main challenge in the connection of the SFC and the heater, was the design of a suitable connecting element. Here, a supersaturated suspension is entering from the SFC and even further cooled as a consequence of the untempered tubing before the heater port. Thus, the risk of wall-crystallization is present, and a preferably short connecting tubing is favorable. A flexible silicone tube with the same diameter $\left(d_{i}=3.18 \mathrm{~mm}\right)$ as the SFC tubing was used during this work. For the connection of the heater and product isolation, the FEP tubing of the heater was extended and connected to the CVSF with a laboratory screw joint.

The CVSF, shown in section III, consisted of two basic elements: tubular, doublewalled cylindrical modules (A, B1, and B2) and a rotating polytetrafluoroethylene (PTFE) screw for axial transport of the particles. To allow visibility inside the apparatus, the modules were made of borosilicate glass with an inner diameter of $25.4 \mathrm{~mm}$ (DN25) and are connected via DN25 flanges with corresponding PTFE seals. The CVSF is continuously filled with suspension entering through the feed inlet in module A. The solid-liquid separation process took place on a porous glass frit (porosity 2, 40-100 $\mu \mathrm{m}, \mathrm{ROBU} \mathrm{GmbH}$ ). The mother liquor left filtration module A by applying vacuum through a vacuum pump (Vacuubrand PC3002 Vario select) to the collection vessel. Simultaneously, the axial transport of the particles to the washing modules B1 and B2 occurred using the PTFE screw. 
These modules were equipped with the same filter medium as Module A. Flat-fan nozzles (Lechler, 610.145) were used for a homogeneous wash liquid distribution in both modules. The wash liquids are pumped with a peristaltic pump (Ismatec Reglo Digital MS-4/12) to the nozzles mounted to the washing modules B1 and B2, respectively, with laboratory screw joints. The corresponding outer jackets and thus the filtrates of module B1 and B2 were separated to prevent back-mixing of the drawn liquid. The washed particles were (pre-) dried in the last module by the drawn air of the vacuum pump and, finally, left the CVSF continuously at the end of module B2 in a beaker. The detailed description of the CVSF dimensions has been described elsewhere [6,15].

\section{Materials and Methods}

\subsection{Substances Used}

As a substance system for the continuous small-scale manufacturing process experiments, the model system L-alanine/water, which has similar particulate properties as high-priced APIs was chosen. L-alanine is a non-toxic and non-essential amino acid biosynthesized in the human primary metabolism. L-alanine was provided by Evonik Industries $A G$ with a purity of $99.7 \%$. As solvent ultrapure, deionized and bacteria-free filtered water (Milli-Q ${ }^{\circledR}$ ) with a total organic carbon-content of maximal 3 ppb was used, purified by a Milli- $\mathrm{Q}^{\circledR}$ Advantage A10 apparatus of Merck KGaA. The solubility curve of L-alanine in water was measured and modeled by WOHLGEMUTH ET AL. [27] (Equation (1)).

$$
c^{*}\left(\mathrm{~g}_{\text {ala }} \mathrm{g}_{\text {solution }}{ }^{-1}\right)=0.11238 \cdot \exp \left(9.0849 \cdot 10^{-3} \cdot \vartheta^{*}\left({ }^{\circ} \mathrm{C}\right)\right),
$$

The density of a saturated L-alanine/water solution at ambient conditions $\left(23^{\circ} \mathrm{C}\right)$ is $1042 \mathrm{~kg} \mathrm{~m}^{-3}$ and the corresponding crystal density of pure L-alanine amounts to $1420 \mathrm{~kg} \mathrm{~m}^{-3}$. As native seed crystals recrystallized, L-alanine particles within a sieve range of 160-200 $\mu \mathrm{m}\left(d_{50}=251.3 \mu \mathrm{m}, d_{90-10}=109.2 \mu \mathrm{m}\right)$ were resuspended in the saturated aqueous solution according to the procedure of OSTERMANN ET AL [28]. The segmentation of the suspension in order to generate the slug flow inside the T-junction was performed by the utilization of synthetic air. For the washing of particles as part of the isolation processing in section III (compare Figure 2), a volumetric mixture of 4:1 of ethanol absolute $(99.9 \%, V W R)$ and water (washing module B1) and pure ethanol absolute (washing module B2) was provided at ambient conditions in order to minimize the agglomeration of product particles and improve washing efficiency, as known from batch experiments [15,29].

\subsection{Experimental Procedure}

Two operating points were set for continuous crystallization (section I Figure 2), which were proven to result in different throughputs, supersaturation levels and product qualities at the end of the SFC [11], in order to demonstrate the operability of connecting to CVSF. In both cases, after the start-up procedure using water-air segmented flow, the seed suspension was fed to the SFC, where dominant crystal growth took part during the cooling process (growth zone). Here, $1 \% \mathrm{~g}_{\text {solid }} \mathrm{g}_{\text {solution }}{ }^{-1}$ recrystallized seed crystals of the sieve fraction $160-200 \mu \mathrm{m}$ was added to a saturated solution $(V=450 \mathrm{~mL})$ at $\vartheta^{*}=50^{\circ} \mathrm{C}$ and homogeneously suspended at a stirring rate of $n=450 \mathrm{rpm}$. Once the seed crystals had been added, after $90 \mathrm{~s}$ the three-way valve below the vessel was turned from a cleaning solution consisting of heated water $\left(\vartheta=50^{\circ} \mathrm{C}\right)$ to sole suspension, so that the suspension arrived at the T-junction. There, the slug formation was performed. The slug length was set by the ratio of the suspension volume flow rate $Q_{\text {susp }}$ and gas volume flow rate $Q_{g a s}$, combined to the total volume flow rate $Q_{t o t}$, according to Equation (2), and was referred to the liquid hold up $\varepsilon_{L, 0}=0.5$.

$$
\varepsilon_{L, 0}=\frac{Q_{\text {susp }}}{Q_{\text {susp }}+Q_{\text {gas }}}=\frac{Q_{\text {susp }}}{Q_{\text {tot }}}
$$


Due to the pressure drop across the tubing depending on the tubing length and the compressibility of the gaseous phase, $Q_{g a s}$ had to be controlled. Prior to this, the synthetic air was heated via a tube-in-tube design, which prevented wall crystallization due to the collision of synthetic air and suspension inside the T-junction. Subsequently, the slugs were cooled down from $\vartheta_{S F C, I N}=50{ }^{\circ} \mathrm{C}$ to $\vartheta_{S F C, O U T}=30{ }^{\circ} \mathrm{C}$ in the growth zone via an applied degressive temperature profile measured by Pt100 temperature sensors (class A; deviation of $\left.0.15+0.002 \cdot \vartheta\left[{ }^{\circ} \mathrm{C}\right]\right)$. On purpose, two challenging operating points of SFC were conducted. By adjusting $Q_{\text {tot }}$ at a constant $\varepsilon_{L, 0}$, the residence time $\tau_{S F C}$, the particles suspension and the cooling rate $\bar{\kappa}$ could be varied. For the case of a low $Q_{t o t}=20 \mathrm{~mL} \mathrm{~min}^{-1}$, $\tau_{S F C}$ of approximately $10 \mathrm{~min}, \bar{\kappa}$ of $1.8 \mathrm{~K} \mathrm{~min}^{-1}$ and a low residual supersaturation at the end of SFC was guaranteed [11]. However, this flow velocity was not sufficient to suspend the particles in a homogeneous state. Accordingly, there was a risk of blocking the heater (section II, Figure 2) due to the residual supersaturation and the settled particles. Therefore, higher $Q_{\text {tot }}$ was considered leading to shorter $\tau_{S F C}(\approx 5 \mathrm{~min})$, higher $\bar{\kappa}\left(=3.6 \mathrm{~K} \mathrm{~min}^{-1}\right)$ and consequently higher residual supersaturation at the end of SFC. The operating parameters for the two-fold determination of each operating point for the continuous crystallization inside the SFC are summarized in Table $1 . Q_{\text {susp }}$ were proven gravimetrically during the start-up procedure.

Table 1. Operating and resulting parameters for continuous crystallization inside the SFC, for the heater and for the particle isolation inside the CVSF in a two-fold determination of each operating point.

\begin{tabular}{|c|c|c|c|}
\hline & Parameters & $\begin{array}{c}\text { CP-Q1.1 and } \\
\text { CP-Q1.2 }\end{array}$ & $\begin{array}{c}\mathrm{CP}-\mathrm{Q} 2.1 \text { and } \\
\mathrm{CP}-\mathrm{Q} 2.2\end{array}$ \\
\hline \multirow{6}{*}{ SFC } & $Q_{t o t}\left[\mathrm{~mL} \min ^{-1}\right]$ & 20 & 40 \\
\hline & $Q_{\text {susp }}\left[\mathrm{mL} \mathrm{min}^{-1}\right]$ & 10 & 20 \\
\hline & $\vartheta_{S F C, I N}\left[{ }^{\circ} \mathrm{C}\right]$ & 50 & 50 \\
\hline & $\vartheta_{S F C, O U T}\left[{ }^{\circ} \mathrm{C}\right]$ & 30 & 30 \\
\hline & $\tau_{S F C}[\mathrm{~min}]$ & 10 & 5 \\
\hline & $\bar{\kappa}\left[\mathrm{K} \min ^{-1}\right]$ & 1.8 & 3.6 \\
\hline \multirow{2}{*}{ Heat. } & $\vartheta_{\text {heater }, T M, I N}\left[{ }^{\circ} \mathrm{C}\right]$ & 63 & 78 \\
\hline & $\vartheta_{\text {heater }, P M, \text { OUT }}\left[{ }^{\circ} \mathrm{C}\right]$ & 39.5 & 43 \\
\hline \multirow{5}{*}{ CVSF } & $n_{\text {screw }}[\mathrm{rpm}]$ & 2 & 2 \\
\hline & $\Delta p_{\text {set }}[\mathrm{mbar}]$ & 400 & 400 \\
\hline & $Q_{\text {wash }}\left[\mathrm{mL} \min ^{-1}\right]$ & 35 & 35 \\
\hline & $\alpha[\%]$ & 6.3 & 12.7 \\
\hline & $\tau_{C V S F, i d}[\mathrm{~min}]$ & 15 & 15 \\
\hline
\end{tabular}

Afterwards, the product suspension was preheated in a vertical-installed tube-in-tube heater (section II, Figure 2) until it entered the undersaturated state and diminished the residual supersaturation. To estimate the process temperature needed after the heater, the relative yield $Y_{\text {rel }}$ (Equation (3)) of crystallization step was required. The respective concentrations c was determined gravimetrically, whereas $c^{*}$ was calculated according to Equation (1).

$$
Y_{r e l, S F C}=\frac{c\left(\vartheta_{S F C, I N}\right)-c\left(\vartheta_{S F C, O U T}\right)}{c\left(\vartheta_{S F C, I N}\right)-c^{*}\left(\vartheta_{S F C, O U T}\right)}
$$

For this purpose, batch experiments were carried out to approximate the final concentration $c\left(\vartheta_{S F C, O U T}\right)$ and associated $Y_{\text {rel }}$ at comparable operating parameters for continuous process CP-Q1 (see Section 4.1.2). For CP-Q2, the $Y_{\text {rel }}$ was approximated by the literature data [11]. This ensured that, after passing the heater, the product suspension was slightly undersaturated and, consequently, the risk of clogging in the filtration, washing, and drying step was minimized. By this procedure, a continuous operation of the further isolation was expected, independent of the existing residual supersaturation from the continuous crystallization. 
Based on the operating parameters of the SFC and calculated CVSF-inputs, the operating parameters of the CVSF were set to maximize the CVSF-performance by means of internal parameters summarized in Table 1 (detailed explanation was shown in our previous studies $[6,15]$ and in the supporting information, Table S1). In the first module of CVSF, the filtration took place. Here, regarding the filling degree $\alpha$ as a crucial internal parameter for CVSF-performance, the CVSF was recommended to operate above $50 \%$ to minimize slip between screw and corpus $[6,15]$. To adjust $\alpha$ at defined inputs $\left(Q_{\text {susp }}, w_{s}\right)$ set by the crystallization process, the rotational screw speed $n_{\text {screw }}$ was adapted [6]. However, with the current design of the CVSF built with a constant screw geometry, the ideal residence time $\tau_{C V S F, i d}$ was proportionally coupled to $\alpha$. This means a decrease in $n_{\text {screw }}$ leads to an increase in $\alpha$ and, simultaneously, to an increase in residence time $\tau_{\mathrm{CVSF}}$, resulting in a longer time for reaching steady-state filtration. For the connection of continuous crystallization and product isolation, a tradeoff between an acceptable $\alpha$ and $\tau_{\text {CVSF }}$ was made, caused by the low $Q_{\text {susp }}$ and $w_{s}$. Therefore, $n_{\text {screw }}$ was set to its actual lowest limit of $2 \mathrm{rpm}$, resulting in an $\tau_{C V S F, \text { id }}=15 \mathrm{~min}$. The subsequent washing zone consists of two modules in which the filter cake was washed at $Q_{\text {wash }}=35 \mathrm{~mL} \mathrm{~min}^{-1}$ and the remaining mother liquor was displaced. Due to the air streams drawn into the washing module B2 by the vacuum pump, an additional drying module was not required. The product particles leaving the CVSF were collected and analyzed, described in the following.

\subsection{Analytics}

To verify the modification of particle characteristics originating from seed crystals by means of continuous crystallization and subsequent obtaining constant product qualities within the product isolation, both the liquid and solid phases were examined at the feed suspension vessel, after the SFC and after the CVSF. This includes the determination of $Y_{\text {rel }}$ by means of gravimetric concentration measurements of the liquid phase before and after crystal growth, the PSDs of seed crystals and product particles, and the determination of residual moisture of product particles after the CVSF.

The liquid solute's concentration is monitored and evaluated gravimetrically with the loss-on-drying method. Therefore, three samples of approximately $6 \mathrm{~g}$ suspension were collected at the feed vessel and after the SFC, filtered by a syringe filter (Chromafil Xtra PTFE, pore size $0.45 \mu \mathrm{m}$, Macherey-Nagel GmbHCo.KG) to ensure particle-free solution, and weighted. After the drying process comprising a first pre-drying in a drying oven for $24 \mathrm{~h}$ at $\vartheta=50^{\circ} \mathrm{C}$ and further drying in a vacuum drying oven (Thermo Heraeus, type Vacutherm VT $6060 \mathrm{M})$ at $\vartheta=50^{\circ}$ for $48 \mathrm{~h}$, the sample concentration was calculated as described in our previous study [6].

For the particle size analysis, the dynamic image analysis sensor QICPIC, equipped with the liquid dispersion system LIXELL, of Sympatec GmbH was used. Therefore, at the respective sampling locations, dry particles (seed crystals \& product particles after CVSF) or suspension (after SFC) were collected ( $>1000$ particles) in excess of tempered saturated aqueous solution to sufficiently dilute potential local supersaturation and thus prevent a potential change after sample drawing. Afterwards, the sample was transferred to a stirred vessel $(n=280 \mathrm{rpm})$ directly connected to the QICPIC measuring cell. By the application of a peristaltic pump (Watson Marlow, $d_{i}=6 \mathrm{~mm}$, Pharmed) installed behind the flow cell to avoid solid attrition or breakage before measuring, the suspension was pumped through the cell (gap: $2 \mathrm{~mm} ; 1024 \times 1024$ pixel resolution; module M6) and analyzed by the laser sensor. The image acquisition and quantification of PSD were executed by artificial neural networks implemented in MATLAB [30,31]. Furthermore, the distinction of single and non-single (aggregated \& agglomerated) crystals as subpopulations of the overall PSD via particle classifier was made [30,31]. In this case, collectives of primary particles arranged and connected via crystal bridges starting from a supersaturated suspension was named agglomerate, while, in the case of aggregates, weak interactions at the edges of the particles could be cemented together to form an agglomerate or could be disrupted again [32]. For the evaluation of the agglomeration behavior during crystallization and 
subsequent filtration, the individual subpopulations of single and non-single crystals, as well as the number-based fraction of aggregates/agglomerates related to the overall number of particles denoted as agglomeration degree $\mathrm{Ag}_{0}$, were considered [31]. The volume-based PSDs were evaluated by means of changes in characteristic size $\Delta d_{50,3}=d_{50,3, \text { OUT }}-d_{50,3, \text { IN }}$ and width of distribution $\Delta d_{90-10,3}=d_{90-10,3, \text { OUT }}-d_{90-10,3, I N}$.

The residual moisture determination was performed analogously to the concentration measurements via the loss-on-drying method, except we used a $1 \mathrm{~g}$ filter cake sample size extracted after the CVSF, as described in detail in our previous studies $[6,15]$.

\section{Results and Discussion}

The first section (Section 4.1) showed the results on the fundamental operability of the integrated process, while the second section (Section 4.2) focused on product quality regarding PSD and residual moisture.

\subsection{Focussing Operability of Integrated Process \\ 4.1.1. Preservation of PSD in Heating Section}

In order to prove obtained consistent particle properties defined in the continuous crystallization process step, preliminary investigations in the heater and the CVSF were conducted. Therefore, a suspension was first prepared in the feed vessel $(\mathrm{V}=450 \mathrm{~mL})$ in a saturated state at room temperature $\left(\vartheta_{\text {heater, }, P M, I N} \approx 23^{\circ} \mathrm{C}\right)$ and directly connected to the heating zone II (compare Figure 2). In the heater, the suspension was then rapidly undersaturated within $\tau_{\text {heater }} \approx 2 \mathrm{~s}$ by $7 \mathrm{~K}\left(\vartheta_{\text {heater,PM,OUT }}=30^{\circ} \mathrm{C}\right)$ and samples were taken before $\left(\right.$ Heater $\left._{\text {IN }}\right)$ and after the heater (Heater OUT $)$, and at the CVSFs outlet (CVSF OUT $)$. To evaluate potential changes in the PSD, four experiments at the same conditions were performed using particles with the desired product particle size (sieve fraction 355-560 $\mu \mathrm{m}$ ) $[6,11]$. The averaged volume-based PSDs and corresponding characteristic values of the experiments are shown in Figure 3.
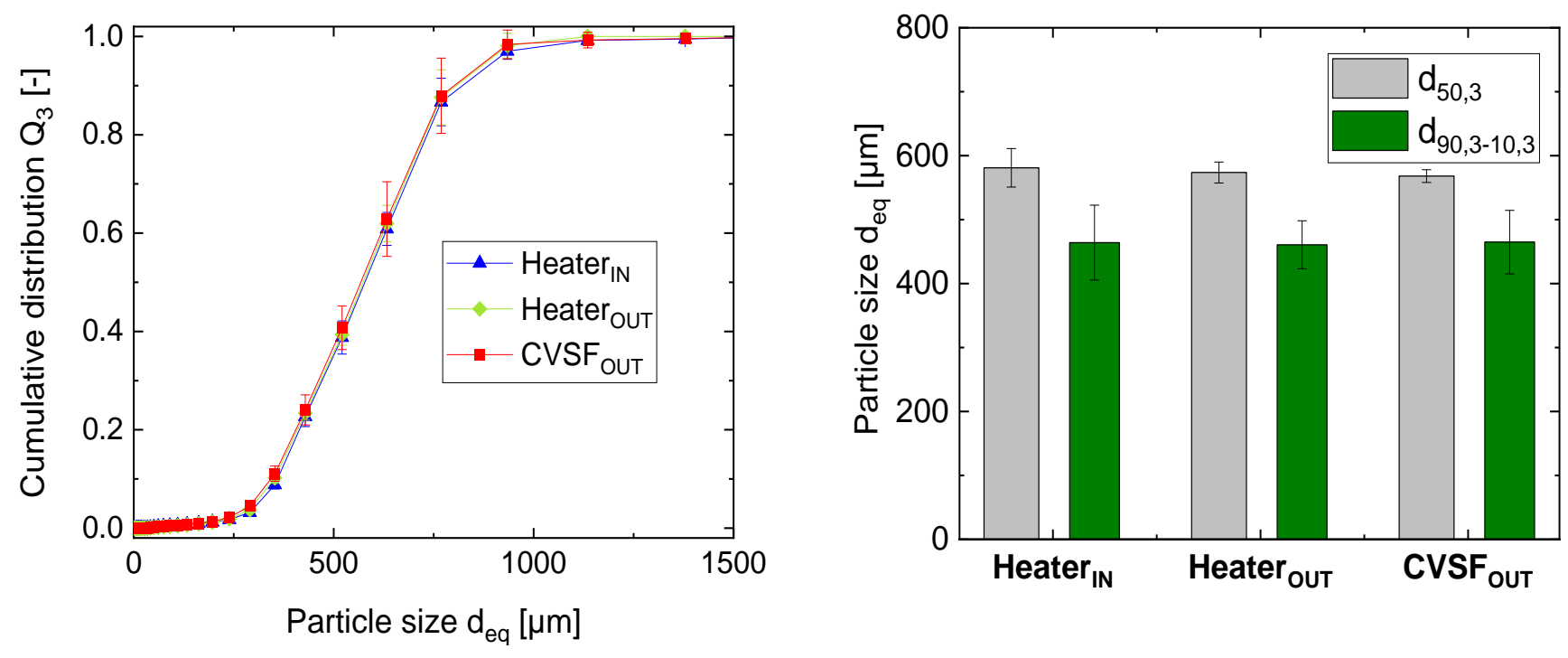

Figure 3. Preliminary experiments (averaged results from four experiments) regarding the heating section using a defined undersaturation: Averaged volume-based PSDs (left) and their characteristic values $d_{50,3}$ and $d_{90,3-10,3}$ (right) at the corresponding sections; Experimental conditions: $\vartheta_{\text {heater, } P M, I N}=23{ }^{\circ} \mathrm{C}$ (saturated suspension), $\vartheta_{\text {heater }, P M, O U T}=30{ }^{\circ} \mathrm{C}$ (undersaturated), $Q_{\text {susp }}=60 \mathrm{~mL} \mathrm{~min}^{-1}, w_{s}=6 \mathrm{wt} . \%, n_{\text {screw }}=3 \mathrm{rpm}$.

No significant change of the PSD was obtained by rapid heating. Furthermore, the PSDs were analyzed regarding a characteristic size $\left(d_{50,3}\right)$ and the characteristic width $\left(d_{90,3-10,3}\right)$, validating the full preservation of PSD. Thus, the entering PSD was preserved throughout the whole process (Heater IN $_{4} \leftrightarrow$ Heater $\left._{\mathrm{OUT}} \leftrightarrow \mathrm{CVSF}_{\mathrm{OUT}}\right)$. Here, it was addition- 
ally shown that neither dissolution nor breakage occurs in the CVSF, which is caused by the short solid-liquid separation time [6,15].

\subsubsection{Estimation of Supersaturation Level after Crystallization}

It is worth mentioning that the figures of $Y_{r e l}$ and $w_{s}$ could only be calculated after the experiment (at least $72 \mathrm{~h}$ ), but nonetheless present essential parameters to choose a sufficient preheating $\left(\vartheta_{\text {heater,PM,OUT }}\right)$ and $n_{\text {screw }}$ of CVSF. $Y_{\text {rel }}$ and $w_{s}$ could either be empirically approximated from batch experiments conducted in the feed suspension vessel or applied solely from SFC experiments (e.g., the literature data [11]). For experiment $\mathrm{CP}-\mathrm{Q} 1$, the batch approximation was used. Therefore, a saturated aqueous solution was prepared at $\vartheta^{*}=50^{\circ} \mathrm{C}$ and stirred at $n=450 \mathrm{rpm}$. After $1 \mathrm{~h}$ holding time at $\vartheta=55^{\circ} \mathrm{C}$, the temperatures were set to $\vartheta=50{ }^{\circ} \mathrm{C}$ and $1 \% \mathrm{~g}_{\text {solid }} \mathrm{g}_{\text {solution }}{ }^{-1}$ recrystallized seed crystals of the sieve fraction $160-200 \mu \mathrm{m}$ from the same basic population are added, and the suspension is cooled down to $\vartheta_{S F C, O U T}=30^{\circ} \mathrm{C}$ with $\bar{\kappa}=1.8 \mathrm{~K} \mathrm{~min}^{-1}$. Concentration samples are taken at regular intervals of approximately $5 \mathrm{~K}$. The results are shown as squared orange points in comparison to the results of the corresponding continuous process in the SFC in the concentration over temperature diagram in Figure 4.

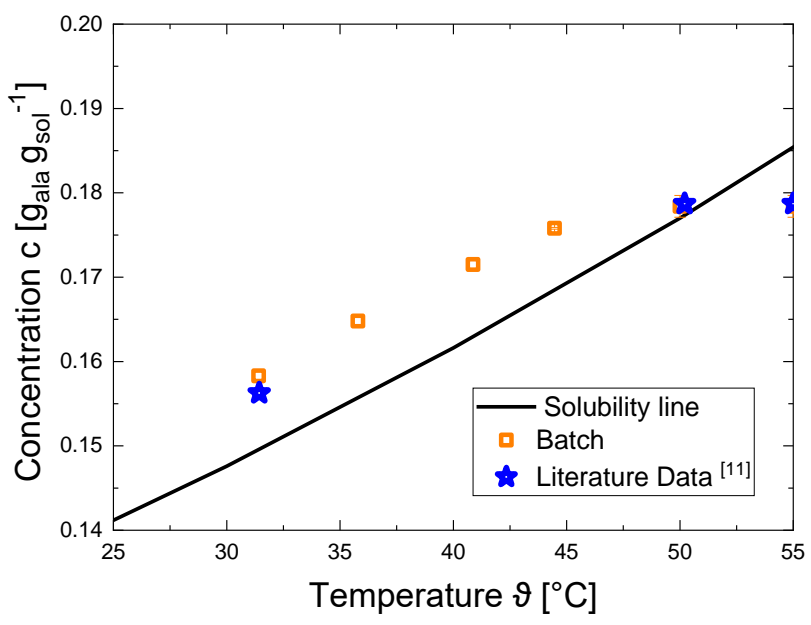

Figure 4. Solution concentrations of batch experiments (orange squares) compared to solution concentrations of continuous SFC operation from literature [11] (blue stars) with constant operating parameters: $\vartheta^{*}=50^{\circ} \mathrm{C}, 1 \% \mathrm{~g}_{\text {solid }} \mathrm{g}_{\text {solution }}{ }^{-1}$ recrystallized seed crystals, $\bar{\kappa}=1.8 \mathrm{~K} \mathrm{~min}^{-1}$.

A relative yield $Y_{\text {rel, batch }}$ of $70.2 \pm 1.0 \%$ was achieved. Compared to the literature data of Termühlen et al. [11] (blue stars), under comparable operating conditions but different seed crystal populations, it can be seen that the final concentrations were almost identical $\left(Y_{\text {rel }, \text { SFC }}=76.9 \pm 1.1 \%\right)$. Accordingly, it was proven that the final concentration of batch experiments could be used in order to approximate the end concentration or supersaturation after SFC crystallization, respectively. The final concentration after the SFC for the CP-Q2 experiment was approximated by literature data [11] $\left(Y_{\text {rel, }, C P-Q 2, a p p .}=59.9 \pm 3.7 \%\right)$. For the two operating points conducted in this study, the heater settings $\left(\vartheta_{\text {heater, } T M, I N}, \vartheta_{\text {heater, } P M, O U T}\right)$ could be calculated and adjusted in order to reach an undersaturated suspension after the heater (shown in Table 1).

\subsubsection{Proof of Operability of Integrated Process}

Figure 5 shows the c,T-diagrams for all four experiments conducted. In the upper half, the results, which were estimated directly from the final concentrations from the batch experiments in the previous section (CP-Q1, $\bar{\kappa}=1.8 \mathrm{~K} \mathrm{~min}^{-1}, Q_{\text {susp }}=10 \mathrm{~mL} \mathrm{~min}^{-1}$ ), are shown. It can be seen that the heater slightly undersaturates the process stream in a targeted manner. Slight variations in concentrations can be explained by the stochastic nature of the crystallization process inside the SFC. The corresponding $Y_{r e l, C P-Q 1}$ of $68.9 \pm 1.5 \%$ was 
achieved, qualitatively matching the values obtained in batch experiments (Section 4.1.2).

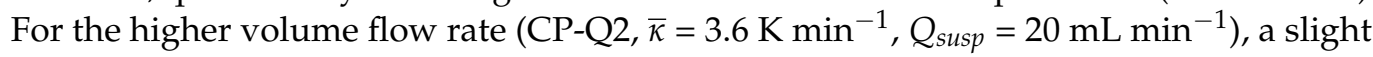
undersaturation was observable in both experiments. However, it could be seen that the targeted undersaturation of $4 \mathrm{~K}$ was not quite achieved, since the final concentration was slightly underestimated, and the $Y_{r e l, C P-Q 2}(44.6 \pm 5.5 \%)$ slightly overestimated.
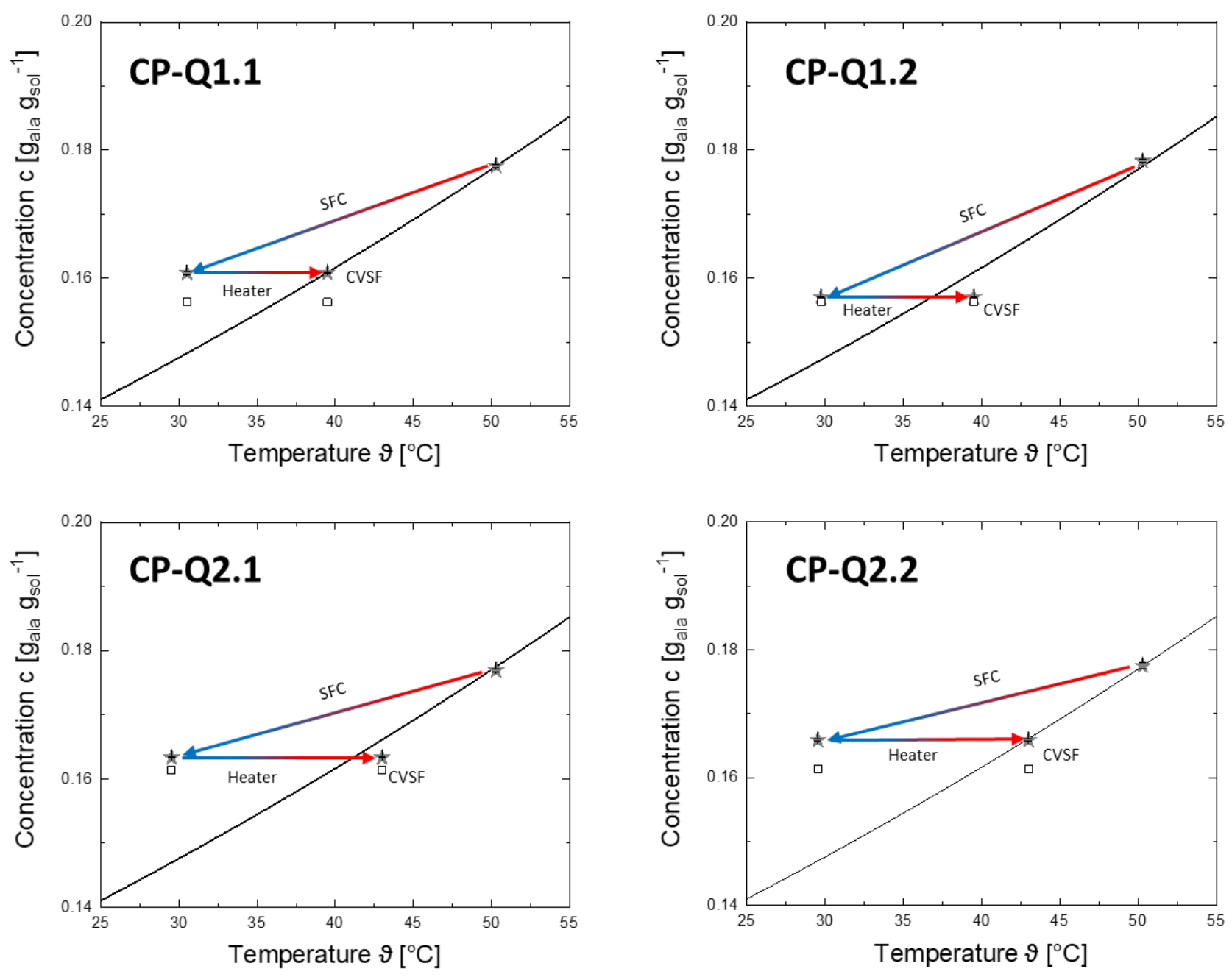

Figure 5. $c, T$ diagrams of the experiments conducted for $Q_{\text {susp }}=10 \mathrm{~mL} \mathrm{~min}^{-1}$ (upper half, CP-Q1) and $Q_{\text {susp }}=20 \mathrm{~mL} \mathrm{~min}^{-1}$ (lower half, CP-Q2). The operating parameters are shown in Table 1. The grey stars show the concentrations measured in the experiments whereas the squares represent the estimated concentrations.

Controlled and defined undersaturation by heating up the process medium leaving the continuous crystallizer could thus be a first step towards fully continuous, integrated processes. Associated therewith, a filtration performance without clogging was expected, and was validated by the measurements of filtrate mass flow rate $\dot{m}$ in the first module, shown in Figure 6. An operation at steady-state conditions for a duration of at least $1 \mathrm{~h}$ could easily be ensured without any clogging of the CVSF. The steady-state operation was validated by the constant $\dot{m}$. Longer operating times would be possible, but were not investigated in detail in the context of this work, since only a limited quantity of seed crystals was available. 


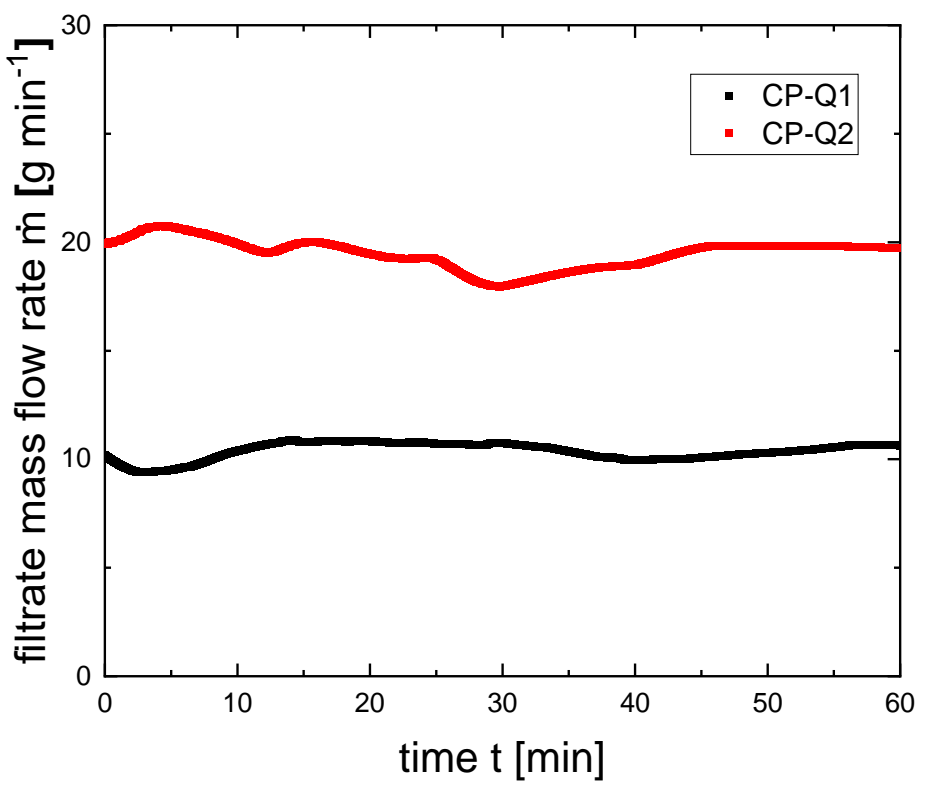

Figure 6. Mass flow rates during the experiment time of the filtrates of module A (filtration module) within the CVSF for experiments CP-Q1 and CP-Q2. $t=0 \mathrm{~min}$ is the time, when the first filtrate is obtained. Operating conditions as shown in Table 1.

Already after approximately $30 \mathrm{~s}$, a constant $\dot{m}$ in module A was observable for both experimental conditions. Slight fluctuations were recognizable for both graphs, which could be explained by fluctuations of the gravimetric recording better than by process variability. Nevertheless, it was clearly visible that $\dot{m}$ for experiment CP-Q2 is almost doubled as for experiment CP-Q1, which fits to the set doubling of $Q_{\text {susp }}$ for crystallization (see Table 1). For the shown experiments, a total mass of approximately $600 \mathrm{~g}$ solvent and $24 \mathrm{~g}$ product particles (CP-Q1) and $1200 \mathrm{~g}$ solvent and $42 \mathrm{~g}$ product particles (CP-Q2) was processed.

It is worth noting that, due to the limited size of the suspension vessel $(450 \mathrm{~mL})$, multiple refilling of the suspension vessel is needed when half of the total volume is reached. Therefore, a segmented flow of Milli- $Q^{\circledR}$ water and air is pumped through the SFC for a short time to allow for the manual refilling of the vessel. Reaching the CVSF, partial dissolution of the particles possibly attached to the filter frit was caused by the water. From this procedure, a positive effect on the integrated process could be derived. A self-induced gradually CIP was performed by solvent rinsing through the filter frit. Although the refilling gap with cleaning solvent was unique for the SFC, a similar strategy could also be applied to different integrated manufacturing processes to benefit from the effects mentioned above.

\subsection{Focusing Product Quality of Integrated Process}

Figure 7 shows the results of the averaged overall PSDs (left) of the seed crystals (seeds), after the SFC (SFC ${ }_{\mathrm{OUT}}$ ) and after the CVSF (CVSF ${ }_{\mathrm{OUT}}$ ) as well as the corresponding subpopulations (right) of single crystals (sc) and non-single crystals (agg). 

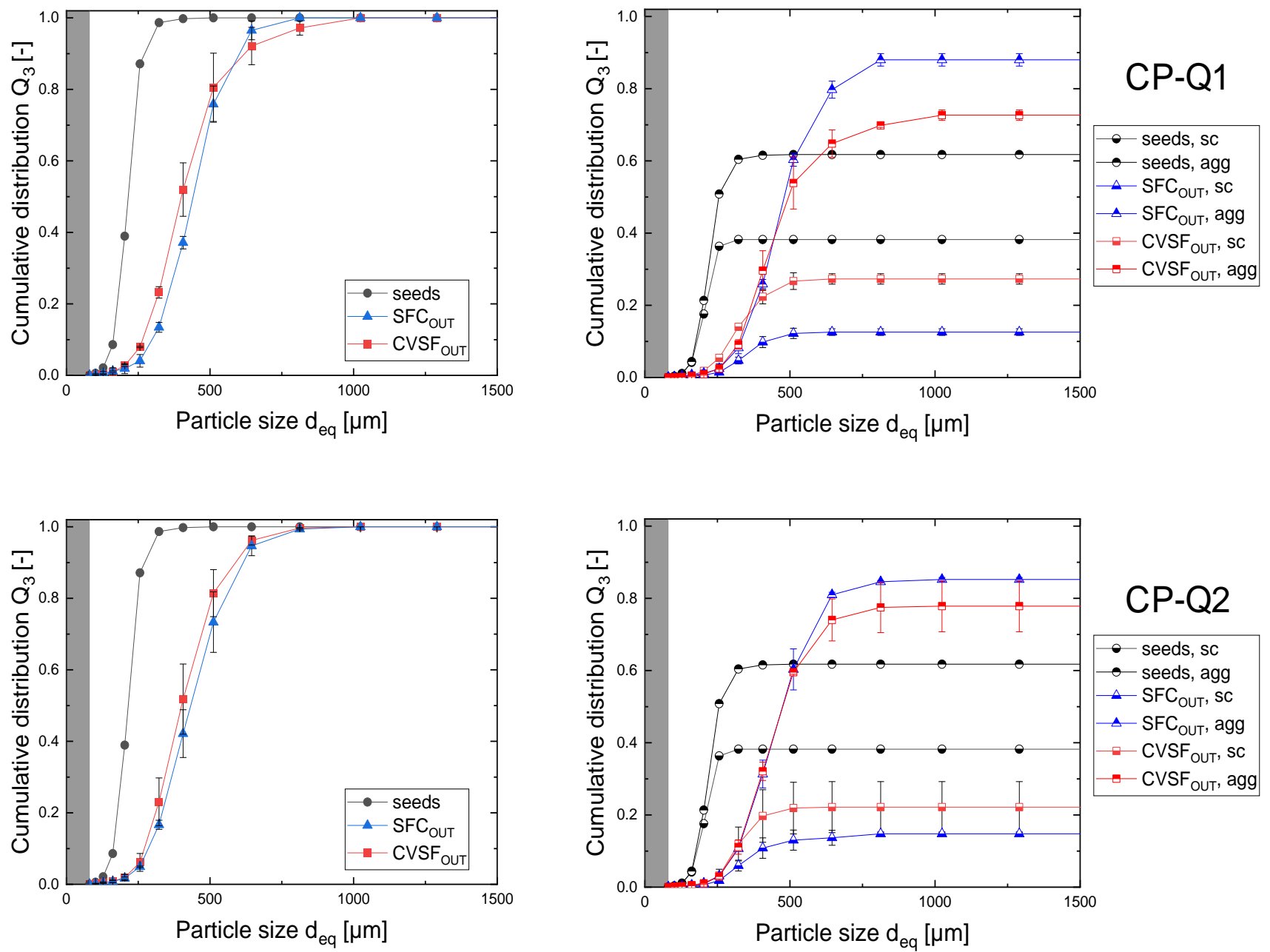

Figure 7. Overall PSDs (left) and corresponding subpopulations of single crystals (sc) and non-single crystals (agg). The upper diagrams show the PSDs for the experiments CP-Q1 and the lower for the experiments CP-Q2. The grey area shows the analyzation limit for particle classification of $80.6 \mu \mathrm{m}$ [30]. Operating conditions as shown in Table 1. Results of the individual experiments are is shown in Table S2.

The superior aim of the experiments conducted was the analysis of the PSD throughout the integrated process. In general, a significant shift towards larger particles was reached for both $Q_{\text {susp }}$ of the SFC (CP-Q1: $\Delta d_{50,3}=226.5 \pm 7.8 \mu \mathrm{m}$; CP-Q2: $\Delta d_{50,3}=218.4 \pm 24.2 \mu \mathrm{m}$ ). Regarding the overall PSDs (left side of Figure 7), it was visible that the PSD after the SFC

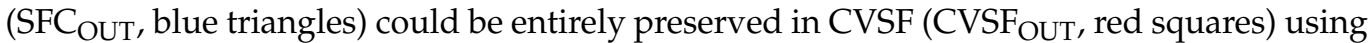
filtration and two-stage washing. The desired particle design of crystal growth in SFC and preservation of PSD in product isolation was successfully shown for varying throughputs and remaining supersaturation levels at the end of SFC. The two right diagrams show the fractions of single crystals (sc) and non-single crystals (agg) at the respective process sections. Here, it was desirable to obtain exclusively crystal growth and to avoid agglomeration. However, a significant decrease of single crystal fraction, and thus an increase in agglomeration/aggregation, was visible. A high increase in agglomeration degree during crystallization was observed for both conditions, indicating insufficient particle suspension. The agglomeration degree of all samples taken after the SFC is higher than the product particles after CVSF, which was also visible in the exemplary binary images in Figure 8. Possible reasons for this deviation may be found in desaggregation in CVSF, which was already observed in a few experiments of the first study about the sole operation of CVSF [6]. Moreover, the two samples (SFC ${ }_{\text {OUT }}$ and $\mathrm{CVSF}_{\mathrm{OUT}}$ ) of each experiment are taken at different times in terms of material traceability. Because of the segmented flow, no back mixing occured in the SFC, whereas a significant back mixing was present in the CVSF 
for the investigated operating conditions. After the CVSF, several particles coming from slugs of various time points were unified in the product particle sample due to the back mixing behavior. This was caused by the non-optimal, low $\alpha$ (see Table 1), mandatorily chosen decision to operate with a suitable $\tau_{C V S F, \text { id }}=15 \mathrm{~min}$. It should be added here that a reduction in the fraction of non-single crystals with no discernible abrasion or breakage (see Figure 8) can be regarded as a major advantage of CVSF for particle isolation.

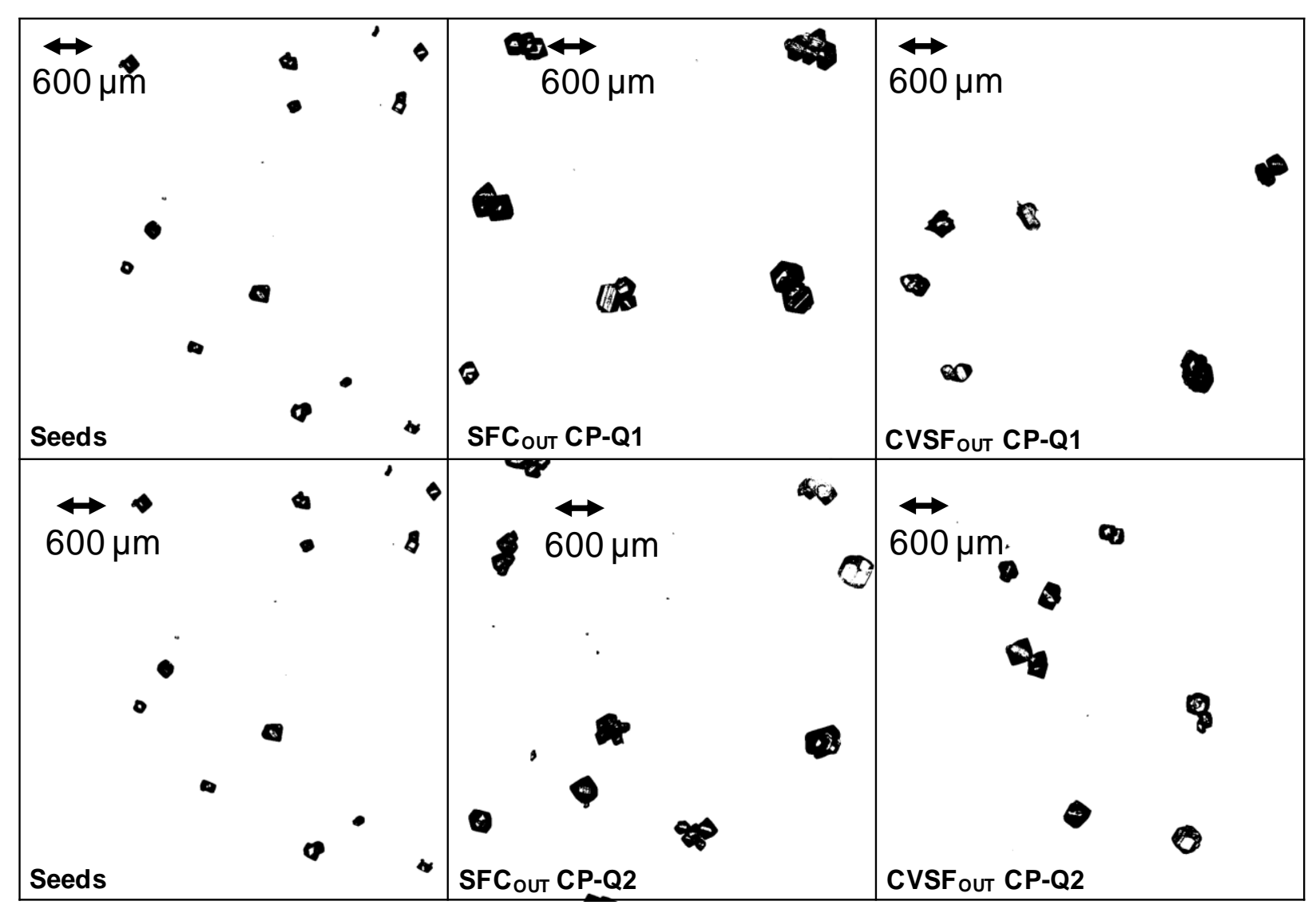

Figure 8. Exemplary QICPIC binary images, (upper half) experiment CP-Q1 and (lower half) experiment CP-Q2. (Left) Seeds, (middle) after the SFC, and (right) after the CVSF.

The residual moisture shows average values of $1.6 \pm 1.1 \%$ (CP-Q1) and $2.7 \pm 2.1 \%$ (CP-Q2). These values are in good agreement with data measured in our previous study for the modular CVSF using two-stage washing [15]. Almost dry, completely isolated and free-flowing product particles leave the CVSF at the outlet. A corresponding video of the particles uniformly leaving the CVSF is given in the supporting information (Video S1). The reduction of the residual moisture to approximately $2 \%$ can be explained by the corresponding air flow through the product particles with the air drawn into the CVSF by the vacuum pump in module B2. Even if $\alpha$ is not optimal for deliquoring, $\tau_{C V S F}$ sufficient to reduce the corresponding residual moisture. The use of ethanol absolute as wash liquid in the second washing module strongly promotes deliquoring due to the higher vapor pressure and lower evaporation enthalpy compared to water (mother liquor). For varying material systems using other wash liquids, an additional drying module could be used to ensure dry particles [23].

\section{Conclusions and Outlook}

Enhanced product quality is possible by the process integration of continuous crystallization with subsequent product isolation. Therefore, in this study, a slug-flow crystallizer (SFC) was combined with the continuous vacuum screw filter (CVSF) for particle design 
throughout the crystal process chain, for finally achieving free-flowing particles with the desired particulate properties. A tube-in-tube heater between SFC and CVSF was implemented and characterized to realize the degradation of remaining supersaturation after crystallization to prevent filter clogging in CVSF. Due to a rapid heating and filtration afterwards, dissolution of particles in the resulting undersaturated suspension and therewith reduced yield and particle sizes were avoided. The supersaturation level at the crystallizer's outlet was estimated by subsequent batch crystallization experiments with the same $\bar{\kappa}$.

A significant shift towards larger particles $\left(\Delta d_{50,3} \approx 200-250 \mu \mathrm{m}\right)$ was reached for both $Q_{\text {susp }}\left(10\right.$ and $\left.20 \mathrm{~mL} \mathrm{~min}^{-1}\right)$ in SFC. However, the fraction of non-single crystals increased in SFC, caused by insufficient suspension behavior. Despite bad conditions in the crystallizer, the PSD was successfully preserved during product isolation using filtration and two-stage washing in CVSF, showing its robust applicability for a wide range of crystallization conditions. The almost dry, free-flowing product particles leave the CVSF continuously with residual moistures of approx. $2 \%$. As the RTD of both individual apparatuses were known, a combined RTD measurement was omitted in this study.

Seeded continuous crystallization and product isolation using L-alanine/water as a model system could enable a steady-state operation over $1 \mathrm{~h}$ for both operating conditions investigated without any filter clogging. Longer operating times are possible for this integrated setup, but were not investigated, in order to save material and resources. A reproducible continuous nucleation unit was planned for the future, so that longer operating times can also be achieved. With the systematic experimental investigations in this study, a remarkable contribution towards intensified and integrated primary manufacturing processes has been made. The salient features of reproducible and enhanced product quality control in SFC, combined with high-quality particle isolation using CVSF, enable a significant advantage towards the industrial applicability of end-to-end continuous manufacturing processes.

\section{Patents}

The CVSF was patented in 2021 as "Rotating-Screw Drying Reactor" as WO 2021/148108 A1.

Supplementary Materials: The following are available online at https:/ /www.mdpi.com/article/ 10.3390/pr9122187/s1, Table S1: Operating parameters of integrated process, Table S2: Results of the integrated process, Figure S1: Experimental setup of integrated process, Video S1: SolidDischarge.mp4.

Author Contributions: Conceptualization, C.S., A.C.K. and K.W.; methodology, C.S., A.C.K. and K.W.; investigation, J.H, A.C.K. and C.S.; writing-original draft preparation, C.S and A.C.K. (equal authorship); writing-review and editing, K.W.; visualization, C.S., A.C.K. and J.H.; supervision, K.W.; project administration, K.W.; funding acquisition, C.S and K.W. All authors have read and agreed to the published version of the manuscript.

Funding: This research work was funded by the state of North-Rhine Westphalia (NRW) and the European Regional Development Fund (EFRE), Project “CLIB-Competence Center Biotechnology (CKB)" (Grant EFRE-0300098) and Project “NRW Patent Validation Program” (Grant EFRE-0400357).

Institutional Review Board Statement: Not applicable.

Informed Consent Statement: Not applicable.

Acknowledgments: The authors express their special thanks to Daniela Ermeling for the technical support during the experiments.

Conflicts of Interest: The authors declare no conflict of interest. The funders had no role in the design of the study; in the collection, analyses, or interpretation of data; in the writing of the manuscript; or in the decision to publish the results. 


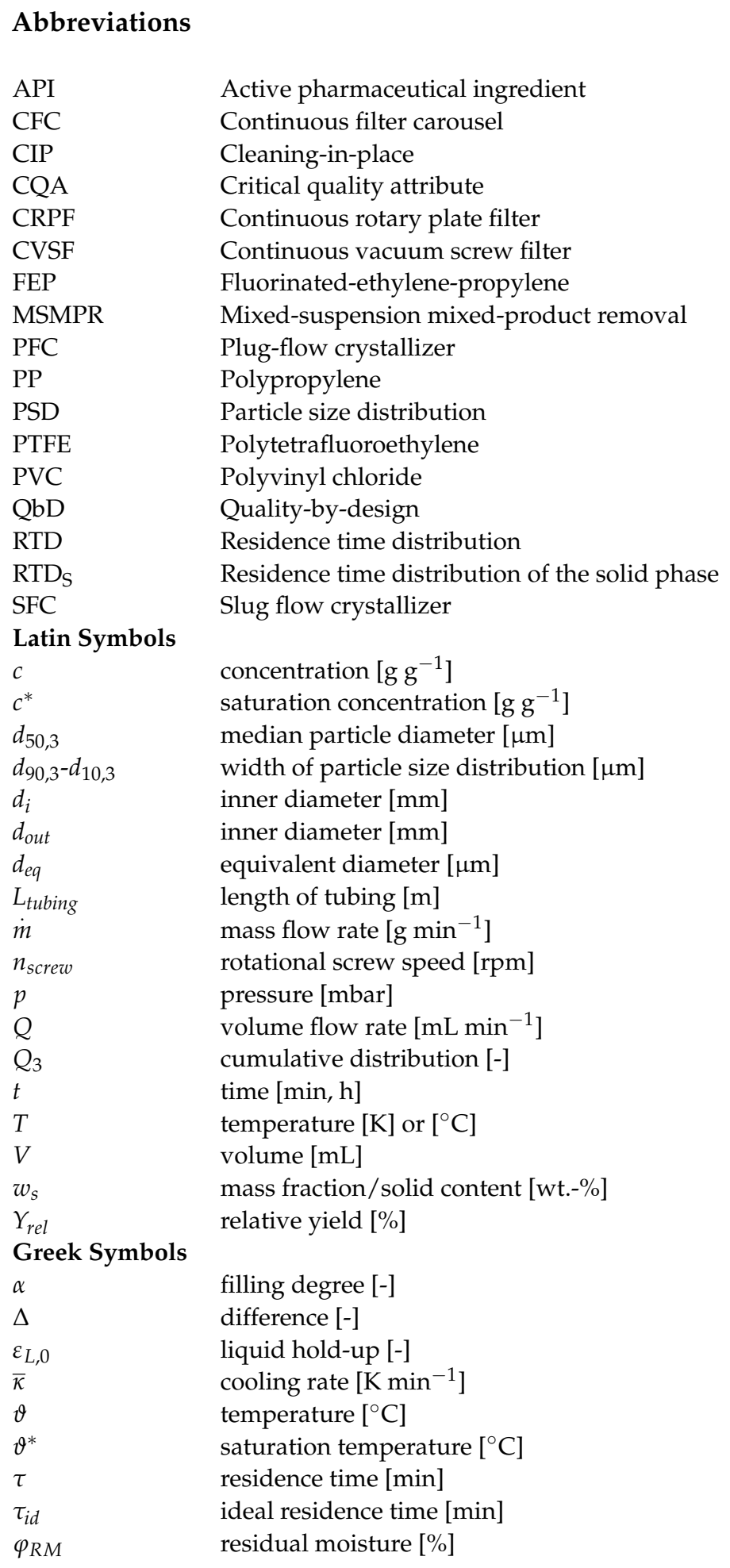

\section{References}

1. Vetter, T.; Burcham, C.; Doherty, M.F. Regions of attainable particle sizes in continuous and batch crystallization processes. Chem. Eng. Sci. 2014, 106, 167-180. [CrossRef]

2. Khinast, J.; Rantanen, J. Continuous Manufacturing of Pharmaceuticals; John Wiley \& Sons, Ltd.: Chichester, UK, 2017.

3. Chen, J.; Sarma, B.; Evans, J.M.B.; Myerson, A.S. Pharmaceutical Crystallization. Cryst. Growth Des. 2011, 11, 887-895. [CrossRef]

4. Orehek, J.; Teslić, D.; Likozar, B. Continuous Crystallization Processes in Pharmaceutical Manufacturing: A Review. Org. Process. Res. Dev. 2021, 25, 16-42. [CrossRef]

5. Cote, A.; Erdemir, D.; Girard, K.P.; Green, D.A.; Lovette, M.A.; Sirota, E.; Nere, N.K. Perspectives on the Current State, Challenges, and Opportunities in Pharmaceutical Crystallization Process Development. Cryst. Growth Des. 2020, 20, 7568-7581. [CrossRef] 
6. Steenweg, C.; Seifert, A.I.; Schembecker, G.; Wohlgemuth, K. Characterization of a Modular Continuous Vacuum Screw Filter for Small-Scale Solid-Liquid Separation of Suspensions. Org. Process. Res. Dev. 2021, 25, 926-940. [CrossRef]

7. Testa, C.J.; Shvedova, K.; Hu, C.; Wu, W.; Born, S.C.; Takizawa, B.; Mascia, S. Heterogeneous Crystallization as a Process Intensification Technology in an Integrated Continuous Manufacturing Process for Pharmaceuticals. Org. Process. Res. Dev. 2021, 25, 225-238. [CrossRef]

8. Domokos, A.; Nagy, B.; Szilágyi, B.; Marosi, G.; Nagy, Z.K. Integrated Continuous Pharmaceutical Technologies-A Review. Org. Process. Res. Dev. 2021, 25, 721-739. [CrossRef]

9. Wood, B.; Girard, K.; Polster, C.S.; Croker, D. Progress to Date in the Design and Operation of Continuous Crystallization Processes for Pharmaceutical Applications. Org. Process. Res. Dev. 2019, 23, 122-144. [CrossRef]

10. Zhang, D.; Xu, S.; Du, S.; Wang, J.; Gong, J. Progress of Pharmaceutical Continuous Crystallization. Engineering 2017, 3, 354-364. [CrossRef]

11. Termühlen, M.; Etmanski, M.M.; Kryschewski, I.; Kufner, A.C.; Schembecker, G.; Wohlgemuth, K. Continuous slug flow crystallization: Impact of design and operating parameters on product quality. Chem. Eng. Res. Des. 2021, 170, 290-303. [CrossRef]

12. Wang, T.; Lu, H.; Wang, J.; Xiao, Y.; Zhou, Y.; Bao, Y.; Hao, H. Recent progress of continuous crystallization. J. Ind. Eng. Chem. 2017, 54, 14-29. [CrossRef]

13. Nagy, Z.K.; El Hagrasy, A.; Litster, J. Continuous Pharmaceutical Processing; Springer International Publishing: Cham, Switzerland, 2020; Volume 42.

14. Yazdanpanah, N.; Nagy, Z.K. The Handbook of Continuous Crystallization; Royal Society of Chemistry: Cambridge, UK, 2020.

15. Steenweg, C.; Seifert, A.I.; Böttger, N.; Wohlgemuth, K. Process Intensification Enabling Continuous Manufacturing Processes Using Modular Continuous Vacuum Screw Filter. Org. Process. Res. Dev. 2021, 25, 2525-2536. [CrossRef]

16. Acevedo, D.; Peña, R.; Yang, Y.; Barton, A.; Firth, P.; Nagy, Z.K. Evaluation of mixed suspension mixed product removal crystallization processes coupled with a continuous filtration system. Chem. Eng. Process. Process. Intensif. 2016, 108, 212-219. [CrossRef]

17. Nagy, B.; Szilágyi, B.; Domokos, A.; Tacsi, K.; Pataki, H.; Marosi, G.; Nagy, Z.K.; Nagy, Z.K. Modeling of pharmaceutical filtration and continuous integrated crystallization-filtration processes. Chem. Eng. J. 2021, 413, 127566. [CrossRef]

18. Domokos, A.; Nagy, B.; Gyürkés, M.; Farkas, A.; Tacsi, K.; Pataki, H.; Liu, Y.C.; Balogh, A.; Firth, P.; Szilágyi, B.; et al. End-to-end continuous manufacturing of conventional compressed tablets: From flow synthesis to tableting through integrated crystallization and filtration. Int. J. Pharm. 2020, 581, 119297. [CrossRef] [PubMed]

19. Liu, Y.C.; Domokos, A.; Coleman, S.; Firth, P.; Nagy, Z.K. Development of Continuous Filtration in a Novel Continuous Filtration Carousel Integrated with Continuous Crystallization. Org. Process. Res. Dev. 2019, 23, 2655-2665. [CrossRef]

20. Mascia, S.; Heider, P.L.; Zhang, H.; Lakerveld, R.; Benyahia, B.; Barton, P.I.; Braatz, R.D.; Cooney, C.L.; Evans, J.M.B.; Jamison, T.F.; et al. End-to-End Continuous Manufacturing of Pharmaceuticals: Integrated Synthesis, Purification, and Final Dosage Formation. Angew. Chem. Int. Ed. 2013, 52, 12359-12363. [CrossRef] [PubMed]

21. Hu, C.; Testa, C.J.; Born, S.C.; Wu, W.; Shvedova, K.; Sayin, R.; Halkude, B.S.; Casati, F.; Ramnath, A.; Hermant, P.; et al. E-factor analysis of a pilot plant for end-to-end integrated continuous manufacturing (ICM) of pharmaceuticals. Green Chem. 2020, 22, 4350-4356. [CrossRef]

22. Steenweg, C.; Schembecker, G.; Wohlgemuth, K. Rotating-Screw Drying Reactor. WO 2021/148108 A1, 29 July 2021.

23. Steenweg, C.; Wohlgemuth, K. Modular, kleinskalig und kontinuierlich-Von der Suspension zum getrockneten Produkt mittels neuartigem Vakuumschraubenfilter. Filtr. Sep. 2021, 4, 4-11.

24. Lührmann, M.-C.; Timmermann, J.; Schembecker, G.; Wohlgemuth, K. Enhanced Product Quality Control through Separation of Crystallization Phenomena in a Four-Stage MSMPR Cascade. Cryst. Growth Des. 2018, 18, 7323-7334. [CrossRef]

25. Termühlen, M.; Strakeljahn, B.; Schembecker, G.; Wohlgemuth, K. Characterization of slug formation towards the performance of air-liquid segmented flow. Chem. Eng. Sci. 2019, 207, 1288-1298. [CrossRef]

26. Su, M.; Gao, Y. Air-Liquid Segmented Continuous Crystallization Process Optimization of the Flow Field, Growth Rate, and Size Distribution of Crystals. Ind. Eng. Chem. Res. 2018, 57, 3781-3791. [CrossRef]

27. Wohlgemuth, K.; Schembecker, G. Modeling induced nucleation processes during batch cooling crystallization: A sequential parameter determination procedure. Comput. Chem. Eng. 2013, 52, 216-229. [CrossRef]

28. Ostermann, M.-C.; Termühlen, M.; Schembecker, G.; Wohlgemuth, K. Growth Rate Measurements of Organic Crystals in a Cone-Shaped Fluidized-Bed Cell. Chem. Eng. Technol. 2018, 41, 1165-1172. [CrossRef]

29. Terdenge, L.-M.; Wohlgemuth, K. Impact of agglomeration on crystalline product quality within the crystallization process chain. Cryst. Res. Technol. 2016, 51, 513-523. [CrossRef]

30. Heisel, S.; Rolfes, M.; Wohlgemuth, K. Discrimination between Single Crystals and Agglomerates during the Crystallization Process. Chem. Eng. Technol. 2018, 41, 1218-1225. [CrossRef]

31. Heisel, S.; Ernst, J.; Emshoff, A.; Schembecker, G.; Wohlgemuth, K. Shape-independent particle classification for discrimination of single crystals and agglomerates. Powder Technol. 2019, 345, 425-437. [CrossRef]

32. Mersmann, A. (Ed.) Crystallization Technology Handbook, 2nd ed.; Dekker: New York, NY, USA, 2001. 